\title{
BMJ Open Heparin-binding protein as a biomarker of gastrointestinal dysfunction in critically ill patients: a retrospective cross-sectional study in China
}

\author{
Jia-Kui Sun, ${ }^{1}$ Xiao Shen, ${ }^{1}$ Xin-Pei Sun, ${ }^{2}$ Xiang Wang, ${ }^{1}$ Wen-Hao Zhang, ${ }^{1}$ \\ Qian-Kun Shi (D) , ${ }^{1}$ Xin-Wei $\mathrm{Mu}^{1}$
}

To cite: Sun J-K, Shen $X$, Sun X-P, et al. Heparin-binding protein as a biomarker of gastrointestinal dysfunction in critically ill patients: a retrospective cross-sectional study in China. BMJ Open 2020;10:e036396. doi:10.1136/ bmjopen-2019-036396

- Prepublication history for this paper is available online. To view these files, please visit the journal online (http://dx.doi. org/10.1136/bmjopen-2019036396).

J-KS and XS contributed equally.

Received 13 December 2019 Revised 18 February 2020 Accepted 14 May 2020

Check for updates

(C) Author(s) (or their employer(s)) 2020. Re-use permitted under CC BY-NC. No commercial re-use. See rights and permissions. Published by BMJ.

${ }^{1}$ Department of Intensive Care Unit, Nanjing First Hospital,

Nanjing Medical University, 68 Changle Road, Nanjing 210006, Jiangsu Province, China

2Department of General Office, Productivity Center of Jiangsu Province, 175 Longpan Road, Nanjing 210042, Jiangsu Province, China

Correspondence to

Dr Qian-Kun Shi;

njdrsqw2019@163.com

\section{ABSTRACT}

Objective To investigate the association of plasma heparin-binding protein (HBP) with the development of acute gastrointestinal injury (AGl) in critically ill patients. Design Clinical retrospective cross-sectional study. Setting A general teaching hospital in China.

Participants Adult patients (age $\geq 18$ years) admitted to our department with an intensive care unit (ICU) stay $\geq 5$ days.

Main outcome measures HBP levels were recorded twice or more within 5 days after admission. The initial AGl grades and the worst AGI grades within 5 days after admission, the number of patients receiving total enteral nutrition (TEN) and the number of patients with feeding intolerance $(\mathrm{FI})$ and with sepsis were also recorded, along with some clinical severity and outcome variables.

Results From June 2018 to May 2019, 221 patients were enrolled in this study. We divided patients into four groups based on the HBP values: $\mathrm{HBP} \leq 20 \mathrm{ng} / \mathrm{mL}, 20<\mathrm{HBP}$ $\leq 50 \mathrm{ng} / \mathrm{mL}, 50<\mathrm{HBP} \leq 100 \mathrm{ng} / \mathrm{mL}$ and $\mathrm{HBP}>100 \mathrm{ng} / \mathrm{mL}$. Significant differences were found in the ratios of AGl deterioration and TEN and the incidence rates of $\mathrm{FI}$ and sepsis among the four groups. Differences were also found among the groups regarding the worst AGI grades. The area under receiver operating characteristic curves for AGI deterioration, severe AGI (grades II or above), TEN and FI were $0.738(p=0.001), 0.774(p<0.001), 0.810(p<0.001)$ and $0.729(p=0.001)$, respectively. The optimal HBP cut-off values for AGI deterioration and severe AGI were $53.27 \mathrm{ng} /$ $\mathrm{mL}$ and $41.26 \mathrm{ng} / \mathrm{mL}$, respectively. However, no differences in ICU duration or 28-day mortality were found.

Conclusions HBP levels were associated with gastrointestinal dysfunction in critically ill patients. Increased HBP was positively correlated with sepsis but it was not correlated with 28-day mortality.

\section{INTRODUCTION}

Gastrointestinal (GI) complications are common and closely related to adverse outcomes in critically ill patients. ${ }^{1-4}$ A recent systematic review and meta-analysis showed that the incidence of GI dysfunction was approximately $40 \%$ and the mortality was $33 \%$ among patients in the intensive care
Strengths and limitations of this study

- This is the first study investigating associations of heparin-binding protein with gastrointestinal dysfunction in critically ill patients.

- The study is single-centre retrospective design and small sample size.

- No patients with acute gastrointestinal injury grade IV were included in this study, and the conclusions might not be applicable to all critically ill patients.

- The results are hypothesis generating. A prospective trial is warranted.

unit (ICU). ${ }^{1}$ As GI failure was considered as 'motor of multiple organ failure', it is important to evaluate GI function as early and accurately as possible. In 2012, the Working Group on Abdominal Problems (WGAP) of the European Society of Intensive Care Medicine (ESICM) developed the definitions and a grading system for acute gastrointestinal injury (AGI) in intensive care patients. ${ }^{3}$ This expert opinion-based AGI grading system was also proven to be a predictor of all-cause mortality in critically ill patients. AGI definitions included primary AGI and secondary AGI. ${ }^{3}$ However, the AGI grading definitions are mainly based on clinical symptoms, but not on objective biomarkers. Moreover, the AGI grades are actually late signs of impaired GI function and cannot adequately reflect the damage of intestinal cells and the mucosal barrier. Therefore, earlier and more sensitive biomarkers are needed to reflect GI function in widespread clinical use.

Heparin-binding protein (HBP), a neutrophil-derived granule protein, was proven to be a useful biomarker for predicting organ dysfunction in sepsis. ${ }^{45}$ Several recent studies found that HBP improved the prediction of acute respiratory distress syndrome (ARDS), circulatory failure and acute kidney 
injury (AKI) in critically ill patients. ${ }^{5-7}$ AGI is considered to be a crucial initial factor for enterogenic infection and multiple organ dysfunction syndrome (MODS). In other words, AGI is also a form of organ dysfunction. In primary AGI, such as abdominal trauma, the neutrophils of damaged intestinal barrier release HBP, and then lead to increase in blood HBP. The increased HBP might impair the intestinal vessels, leading to vascular leakage and tissue oedema, ${ }^{45}$ and then result in enterogenous infection and other organ injury even MODS. In secondary AGI, such as sepsis, bacteria or lipopolysaccharide (LPS) could also stimulate the neutrophils in blood to release HBP, then the increased HBP impair endothelial cell barriers of intestinal vessels, leading to further GI injury. ${ }^{45}$ At the same time, HBP could bind to the LPS, ${ }^{4}$ and it might play an important role in neutralising LPS during intestinal mucosal barrier injury. Furthermore, HBP has a broad spectrum of antimicrobial activity, especially against gram-negative bacteria. ${ }^{4}$ Therefore, the increased HBP level might be an indicator of early AGI. Accordingly, we hypothesised that increased HBP levels were associated with AGI development in critically ill patients, and this study was performed to verify this hypothesis.

\section{MATERIALS AND METHODS \\ Patients}

From June 2018 to May 2019, all adult patients (age $\geq 18$ years) admitted to the surgical ICU of our department, Nanjing First Hospital with an ICU stay $\geq 5$ days were included in this clinical retrospective study. Patients with chronic organ dysfunction (eg, hepatic or renal dysfunction), terminal cancer, coagulation dysfunction, malnutrition or immunodeficiency were all excluded. Figure 1 shows the flow diagram of the participants. All patients received specialised critical care treatments, such as intensive monitoring, oxygen administration or mechanical ventilation, glucose control, fluid resuscitation, vasopressor administration or renal replacement therapy as needed, and antimicrobial therapy.
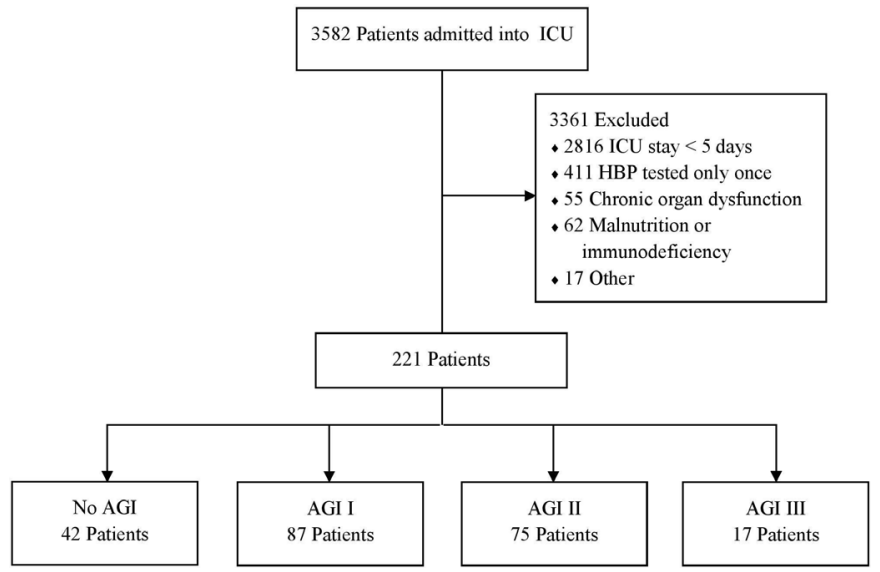

Figure 1 The flow diagram of participants. AGI, acute gastrointestinal injury; HBP, heparin-binding protein; ICU, intensive care unit.

\section{Definitions}

AGI was defined as a malfunction of the GI tract in critically ill patients due to acute illness and was categorised into four grades according to its severity. ${ }^{3}$ Primary AGI was associated with primary disease or direct injury to organs of the GI system, for example, peritonitis, pancreatic or hepatic pathology, abdominal surgery and abdominal trauma. ${ }^{3}$ Secondary AGI developed as the consequence of a host response in critical illness without primary pathology in the GI system, for example, GI malfunction in a patient with pneumonia, cardiac pathology, non-abdominal surgery or trauma. ${ }^{3}$ AGI grade I was defined as an increased risk of developing GI dysfunction or failure (a self-limiting condition); AGI grade II was defined as GI dysfunction (a condition that requires interventions); AGI grade III was defined as GI failure (GI function cannot be restored with interventions) and AGI grade IV was defined as dramatically manifesting GI failure (a condition that is immediately life-threatening). ${ }^{3}$ In this study, we determined AGI grade II or above as severe AGI. Total enteral nutrition (TEN) was defined as full (at least $20 \mathrm{kcal} / \mathrm{kg}$ body weight (BW)/day within 72 hours of feeding ${ }^{39}$ ) enteral nutrition via nasogastric or nasojejunal tube. AGI deterioration was defined as AGI grade from 0 to I/II/III, from I to II/III or from II to III. GI symptoms included nausea, vomiting, diarrhoea, paralysis, high gastric residual volumes, intra-abdominal hypertension and GI bleeding. ${ }^{3}$ Feeding intolerance (FI) syndrome was a general term indicating intolerance of enteral feeding for whatever clinical reasons, and FI was also considered present if at least $20 \mathrm{kcal} / \mathrm{kg} \mathrm{BW} /$ day via the enteral route could not be reached within 72 hours of a feeding attempt. ${ }^{3}$ Sepsis was defined as life-threatening organ dysfunction caused by a dysregulated host response to infection. ${ }^{9}$ The diagnostic criteria of ARDS were in accordance with the Berlin definitions. ${ }^{10}$ The definitions of AKI were based on the 2012 Kidney Disease: Improving Global Outcomes guidelines. ${ }^{11}$ MODS was defined as the combined dysfunction of two or more organs.

\section{Data collection}

The baseline clinical characteristics, including age, sex and causes of ICU admission were recorded on ICU admission. Patients with inflammation, infection, sepsis, trauma, burn, hypotension, shock and organ dysfunction would be considered HBP measurements in our centre. Plasma HBP levels were recorded if they were detected twice or more within 5 days after admission. The acute physiology and chronic health evaluation (APACHE) II score, sequential organ failure assessment (SOFA) score, blood lactate, white blood cell (WBC) count, procalcitonin (PCT) and initial AGI grades at the time of admission were also recorded. The AGI grades were recorded retrospectively based on the electronic nursing score forms in our hospital. The worst AGI grades within 5 days after admission, number of patients with primary AGI or secondary AGI, number of patients receiving TEN and number of patients with FI or sepsis were registered. 
Moreover, the 28-day mortality and duration of the ICU stay were also recorded. The primary end point was the ratio of AGI deterioration, and the secondary end points were the FI incidence rates and TEN ratios within 5 days after admission.

Plasma HBP was detected immediately by the commercially available Axis-Shield HBP microtitre plate ELISA kits using sodium citrate tubes (Becton, Dickinson and Company, Franklin Lakes, New Jersey, USA). Tverring and colleagues ${ }^{5}$ divided $\mathrm{HBP}$ values into four groups: HBP $\leq 10 \mathrm{ng} / \mathrm{mL}, \mathrm{HBP}>10 \leq 20 \mathrm{ng} / \mathrm{mL}, \mathrm{HBP}>20 \leq 40 \mathrm{ng} / \mathrm{mL}$ and HBP $>40 \mathrm{ng} / \mathrm{mL}$ in their study about sepsis-related AKI. Previous studies showed that the optimal cut-off level for HBP in diagnosing sepsis, severe sepsis or septic shock was $\geq 15 \mathrm{ng} / \mathrm{mL}, \geq 28.1$ or $30 \mathrm{ng} / \mathrm{mL}$ and $\geq 103.5 \mathrm{ng} / \mathrm{mL}$, respectively. ${ }^{812} 13$ However, to our knowledge, there are no investigations on the relations between HBP levels and GI function in critically ill patients. The HBP values in our study were relatively large, and the median plasma HBP level was 51.19 (29.99-99.50) ng/mL. Therefore, we divided patients into four groups based on means of the worst values: $\mathrm{HBP} \leq 20 \mathrm{ng} / \mathrm{mL}(\mathrm{n}=38), 20<\mathrm{HBP} \leq 50 \mathrm{ng} /$ $\mathrm{mL} \quad(\mathrm{n}=71), \quad 50<\mathrm{HBP} \leq 100 \mathrm{ng} / \mathrm{mL} \quad(\mathrm{n}=59)$ and $\mathrm{HBP}$ $>100 \mathrm{ng} / \mathrm{mL}(\mathrm{n}=53)$. The patient number of each group was also appropriate.

\section{Patient and public involvement}

This was a retrospective study. No patients were involved in the design, recruitment and conduct of the study. No patients were asked for advice on interpretation of the results. There are no plans to disseminate the findings to study participants.

\section{Statistical analysis}

The Kolmogorov-Smirnov test was first performed to test the normal distribution of the data. Normally distributed data were expressed as the means $\pm \mathrm{SD}$ and were compared by $t$ tests. Abnormally distributed data were expressed as the medians (IQRs) and were compared by the MannWhitney U test or the Kruskal-Wallis test. Categorical variables were presented as absolute numbers or percentages and were analysed using the $\chi^{2}$ test or Fisher's exact test. To take into account the repeated nature of the variables, analysis of variance (ANOVA) for repeated measurements of the general linear model was implemented. To determine the factors associated with severe AGI (grade II or above), we performed a series of several univariate logistic regression analyses using the above-mentioned variables. Variables with $\mathrm{p}<0.1$ in univariate analyses were tested in further multivariate logistic regression analyses. Receiver operating characteristic (ROC) curves were used to evaluate the associations between HBP and AGI, FI, TEN, sepsis and 28-day mortality. IBM Statistical Package for the Social Sciences (SPSS V.22.0) software was used for statistical analysis, and $\mathrm{p}<0.05$ was considered statistically significant. SPSS scatter plots and a correlation analysis were performed to evaluate the relevance between HBP and blood lactate, WBC count and PCT, respectively. The statistical methods of this study were reviewed by Qiao Liu, a biostatistician from the Center for Disease Control and Prevention of Jiangsu Province in China.

\section{RESULTS}

As shown in figure 1, a total of 221 patients were enrolled in this clinical retrospective study during the research period. Only 57 patients $(57 / 221,25.8 \%)$ had no AGI on ICU admission. During ICU stay, 68 patients suffered primary AGI, whereas 96 patients suffered secondary AGI. Four hundred and ninety-four HBP measurements were performed in total, and 166 patients had their ICU admission day values of HBP measured. The mean number of HBP measurements per patient was 2.4. The demographic data and clinical parameters of the patients on admission are presented in table 1. One hundred and seventy-seven patients had undergone surgery, of which 55 patients had undergone abdominal surgery before ICU admission. Eighty-five patients (85/221, 38.5\%) had FI symptoms during enteral feeding, and 157 patients $(157 / 221,71.0 \%)$ received TEN. Fifty-eight patients $(58 / 221,26.2 \%)$ suffered sepsis, and 27 patients $(27 / 221$, $12.2 \%$ ) died of MODS or infectious complications during the hospital stay (within 28 days).

\section{HBP and the variables}

The median plasma HBP levels were $51.19 \mathrm{ng} / \mathrm{mL}$ on ICU admission, and the HBP values were expressed as the means of the recorded results within 5 days after admission. We divided patients into four groups based on the worst HBP values: $\mathrm{HBP} \leq 20 \mathrm{ng} / \mathrm{mL}$ (defined as group A), $20<\mathrm{HBP} \leq 50 \mathrm{ng} / \mathrm{mL}$ (defined as group B), $50<\mathrm{HBP}$ $\leq 100 \mathrm{ng} / \mathrm{mL}$ (defined as group C) and HBP $>100 \mathrm{ng} / \mathrm{mL}$ (defined as group D).

As shown in table 2, significant differences were found in the ratios of AGI deterioration and TEN, and the incidence rates of FI and sepsis among the four groups. There were also significant differences in SOFA scores, PCT, lactate and WBC levels among the four groups. However, no differences were found in APACHE II scores, ICU days and 28-day mortality.

The AGI deterioration and FI incidence rates of group A were significantly lower than those of group $C(p=0.023$ and 0.014 , respectively) or group $\mathrm{D}(\mathrm{p}=0.001$ and 0.001 , respectively). The TEN ratios of group A were significantly higher than those of group $\mathrm{C}(\mathrm{p}=0.034)$ or group $\mathrm{D}(\mathrm{p}<0.001)$. However, no differences in these variables were found between groups A and B or between groups $\mathrm{B}$ and $\mathrm{C}$. The AGI deterioration and FI incidence rates of group B were also significantly lower than those of group $\mathrm{D}$ ( $\mathrm{p}=0.049$ and 0.05 , respectively). The TEN ratios of group B were also significantly higher than those of group D $(p<0.001)$. No differences in the four variables were found except for a difference in the TEN ratios $(\mathrm{p}=0.044)$ between groups $\mathrm{C}$ and $\mathrm{D}$.

The SOFA scores of group $\mathrm{D}$ were significantly higher than those of groups A, B and C $(\mathrm{p}=0.002,0.044$ and 


\begin{tabular}{lc} 
Table 1 & Demographic data and clinical parameters \\
\hline Variables & Values \\
\hline Age (years) & $66(54-76)$ \\
Sex (male:female) & $143: 78$ \\
Causes of ICU admission ( $\mathrm{n}, \%)$ & \\
\hline Abdominal infection & $22(10.0)$ \\
Thoracic/pulmonary infection & $46(20.8)$ \\
Urinary infection & $4(1.8)$ \\
Other infections & $3(1.4)$ \\
AECOPD & $6(2.7)$ \\
Acute kidney injury & $10(4.5)$ \\
ARDS/severe pneumonia & $11(5.0)$ \\
Cerebral haemorrhage/infarction & $13(5.9)$ \\
Multiple trauma & $13(5.9)$ \\
Burn & $4(1.8)$ \\
Haemorrhagic shock & $6(2.7)$ \\
Severe acute pancreatitis & $4(1.8)$ \\
Coronary heart disease & $8(3.6)$ \\
Acute cardiac dysfunction & $17(7.7)$ \\
Aortic dissection & $24(10.9)$ \\
Valvular heart disease & $21(9.5)$ \\
Other & $9(4.1)$ \\
Sepsis (n, \%) & $58(26.2)$ \\
\hline
\end{tabular}

\begin{tabular}{|c|c|}
\hline \multicolumn{2}{|l|}{ Categories (n, \%) } \\
\hline Elective surgery & $116(52.5)$ \\
\hline Emergency surgery & $61(27.6)$ \\
\hline Medical & $44(19.9)$ \\
\hline Pathology (Gl:non-Gl) & $68: 153$ \\
\hline APACHEll scores & $16(13-21.5)$ \\
\hline SOFA scores & $6(4-8)$ \\
\hline HBP levels (ng/mL) & $51.19(29.99-99.50)$ \\
\hline \multicolumn{2}{|l|}{ AGI grades (n, \%) } \\
\hline 0 & $57(25.8)$ \\
\hline 1 & $107(48.4)$ \\
\hline II & 47 (21.3) \\
\hline III & $10(4.5)$ \\
\hline Feeding intolerance (n, \%) & $85(38.5)$ \\
\hline PCT (ng/mL) & $2.51(0.42-10.33)$ \\
\hline WBC $\left(10^{9} / \mathrm{L}\right)$ & $12.25(9.42-15.69)$ \\
\hline Lactate (mmol/L) & $2.20(1.30-3.50)$ \\
\hline ICU days & $7.0(5.0-13.0)$ \\
\hline Death (n, \%) & 27 (12.2) \\
\hline
\end{tabular}

The age, sex, causes of ICU admission, categories, pathology, APACHE II scores, SOFA scores, HBP levels, AGI grades, PCT, WBC and lactate were admission variables. The sepsis and feeding intolerance were summary variables for 5 days. The ICU days and death (28-day mortality) were summary variables for hospital stay. AECOPD, acute exacerbation of chronic obstructive pulmonary disease; AGI, acute gastrointestinal injury; APACHEIl, acute physiology and chronic health evaluation II; ARDS, acute respiratory distress syndrome; GI, gastrointestinal; HBP, heparin-binding protein; ICU, intensive care unit; PCT, procalcitonin; SOFA, sequential organ failure assessment; WBC, white blood cell.
$<0.001$, respectively). The SOFA scores of group B were also higher than those of group $\mathrm{C}(\mathrm{p}=0.029)$. No differences in the SOFA scores were found between groups A and B or between groups A and C. The PCT, lactate and WBC levels in group A were significantly lower than those of group $\mathrm{C}$ ( $\mathrm{p}=0.036,0.002$ and 0.003 , respectively) or group D $(\mathrm{p}<0.001,<0.001$ and 0.001 , respectively); however, no differences in these variables were found between groups $A$ and $B$, with the exception of lactate $(p=0.002)$. The PCT, lactate and WBC levels of group B were also significantly lower than those of group D ( $<<0.001,0.012$ and 0.005, respectively); however, no differences in these variables were found between groups B and C. The PCT $(p<0.001)$ and lactate $(\mathrm{p}=0.002)$ levels of group $\mathrm{C}$ were significantly lower than those of group D. These results indicated that patients with higher HBP levels had worse AGI grades, clinical severity and outcomes.

\section{AGI and the variables}

The worst AGI grades within 5 days after admission were recorded; at the end of the study period, 42 patients $(42 / 221,19.0 \%)$ had no AGI, 87 patients $(87 / 221$, $39.4 \%)$ developed AGI grade I, 75 patients (75/221, $33.9 \%)$ developed AGI grade II and 17 patients (17/221, $7.7 \%$ ) developed AGI grade III. We divided the patients into four groups based on the worst AGI grades: AGI 0 (defined as group A), AGI I (defined as group B), AGI II (defined as group C) and AGI III (defined as group D).

As shown in table 3, significant differences were found in above clinical variables among the four groups. The HBP, PCT and lactate levels of group A were significantly lower than those of group B ( $\mathrm{p}=0.004,0.006$ and 0.003 , respectively). Significant differences were found in the above clinical variables except the WBC levels between groups $A$ and $C(p<0.05)$. Significant differences were also found in the above clinical variables between groups $A$ and $D(p<0.05)$ or between groups B and D $(p<0.01)$. The HBP and PCT levels, FI and sepsis incidence rates, SOFA scores, ICU days and 28-day mortality of group B were significantly lower than those of group $\mathrm{C}(\mathrm{p}<0.05)$. The TEN ratios of group $\mathrm{B}$ were significantly higher than those of group $\mathrm{C}(\mathrm{p}<0.001)$. The HBP, PCT, lactate, WBC levels, SOFA scores and ICU days of group $\mathrm{C}$ were significantly lower than those of group D $(\mathrm{p}<0.05)$.

Moreover, the HBP levels of patients with FI were significantly higher than those of patients without FI (66.78 (36.34-130.31) vs 42.78 (22.08-86.0), $\mathrm{p}=0.001)$, and the HBP levels of patients with TEN were also significantly lower than those of patients without TEN (42.17 (23.96-77.80) vs 88.94 (45.16-164.04), $\mathrm{p}<0.001)$ during the ICU stay. These results indicated that patients with more severe AGI had higher HBP levels and worse clinical severity and outcomes.

\section{Risk model of AGI}

To determine the factors associated with severe AGI (grade II or above), univariate logistic regression was performed using the above-mentioned variables (HBP, 
Table 2 Clinical variables of severity and outcomes

\begin{tabular}{|c|c|c|c|c|c|}
\hline & $\begin{array}{l}\mathrm{HBP} \leq 20 \mathrm{ng} / \mathrm{mL} \\
(\mathrm{n}=38)\end{array}$ & $\begin{array}{l}20<\mathrm{HBP} \leq 50 \mathrm{ng} / \mathrm{mL} \\
(\mathrm{n}=71)\end{array}$ & $\begin{array}{l}50<H B P \leq 100 \mathrm{ng} / \mathrm{mL} \\
(n=59)\end{array}$ & $\begin{array}{l}\text { HBP >100 ng/mL } \\
(n=53)\end{array}$ & $P$ value \\
\hline $\begin{array}{l}\text { AGI deterioration } \\
(\mathrm{n}, \%)\end{array}$ & $5(13.2)$ & $20(28.2)$ & 20 (33.9) & $24(45.3)$ & 0.011 \\
\hline TEN (n, \%) & $33(86.8)$ & $58(81.7)$ & $40(67.8)$ & $26(49.1)$ & $<0.001$ \\
\hline Sepsis (n, \%) & $5(13.2)$ & $16(22.5)$ & $17(28.8)$ & $20(37.7)$ & 0.053 \\
\hline SOFA scores & $6.0(3.75-7.0)$ & $6.0(4.0-9.0)$ & $5.0(4 .-7.0)$ & $7.0(5-10.0)$ & 0.001 \\
\hline ICU days & $7.0(5.0-11.25)$ & $9.0(5.0-14.0)$ & $7.0(5.0-10.0)$ & $9.0(5.0-16.0)$ & 0.187 \\
\hline Death (n, \%) & $5(13.2)$ & $10(14.1)$ & $6(10.2)$ & $6(11.3)$ & 0.912 \\
\hline PCT (ng/mL) & $0.89(0.14-3.53)$ & $1.64(0.18-7.09)$ & $2.77(0.43-7.38)$ & $10.39(2.45-62.22)$ & $<0.001$ \\
\hline $\begin{array}{l}\text { Lactate } \\
(\mathrm{mmol} / \mathrm{L})\end{array}$ & $1.4(0.98-2.20)$ & $2.3(1.3-3.5)$ & $2.1(1.4-3.1)$ & $3.1(2.1-4.5)$ & $<0.001$ \\
\hline
\end{tabular}

The HBP was summary variable for 5 days (mean values). The AGI grades were summary variables for 5 days (the worst values). The FI, TEN and sepsis were summary variables for 5 days. The APACHE II scores, SOFA scores, PCT, lactate and WBC were admission variables. The ICU days and death (28-day mortality) were summary variables for hospital stay.

AGI, acute gastrointestinal injury; APACHEII, acute physiology and chronic health evaluation II; FI, feeding intolerance ; HBP, heparin-binding protein; ICU, intensive care unit; PCT, procalcitonin; SOFA, sequential organ failure assessment; TEN, total enteral nutrition; WBC, white blood cell.

PCT, lactate, WBC, age, APACHEII score, ICU days, sepsis or not and death or not), and variables that showed statistical significance $(p<0.1)$ were identified by further multivariate logistic regression. As shown in table 4, four variables, including HBP, were established as independent risk factors for the development of severe AGI.

The correlations between HBP and the clinical severity variables were also analysed. As shown in figure 2 , the HBP levels were positively correlated with PCT $\left(\mathrm{R}^{2}=0.172\right)$ and lactate $\left(R^{2}=0.118\right)$, and they were weakly correlated with
WBC $\left(\mathrm{R}^{2}=0.09\right)$. No correlations were found between HBP levels and other variables.

The ROC curves were performed to assess the associations between HBP and AGI deterioration ratio, the TEN ratio, FI incidence, sepsis incidence and 28-day mortality in critically ill patients. The area under curves (AUCs) of sepsis, TEN and FI were $0.741 \quad(p=0.001), 0.810(p<0.001)$ and $0.729(\mathrm{p}=0.001)$, respectively. No significant association was found between HBP values and 28-day mortality. Optimal cut-off points for HBP were also derived from

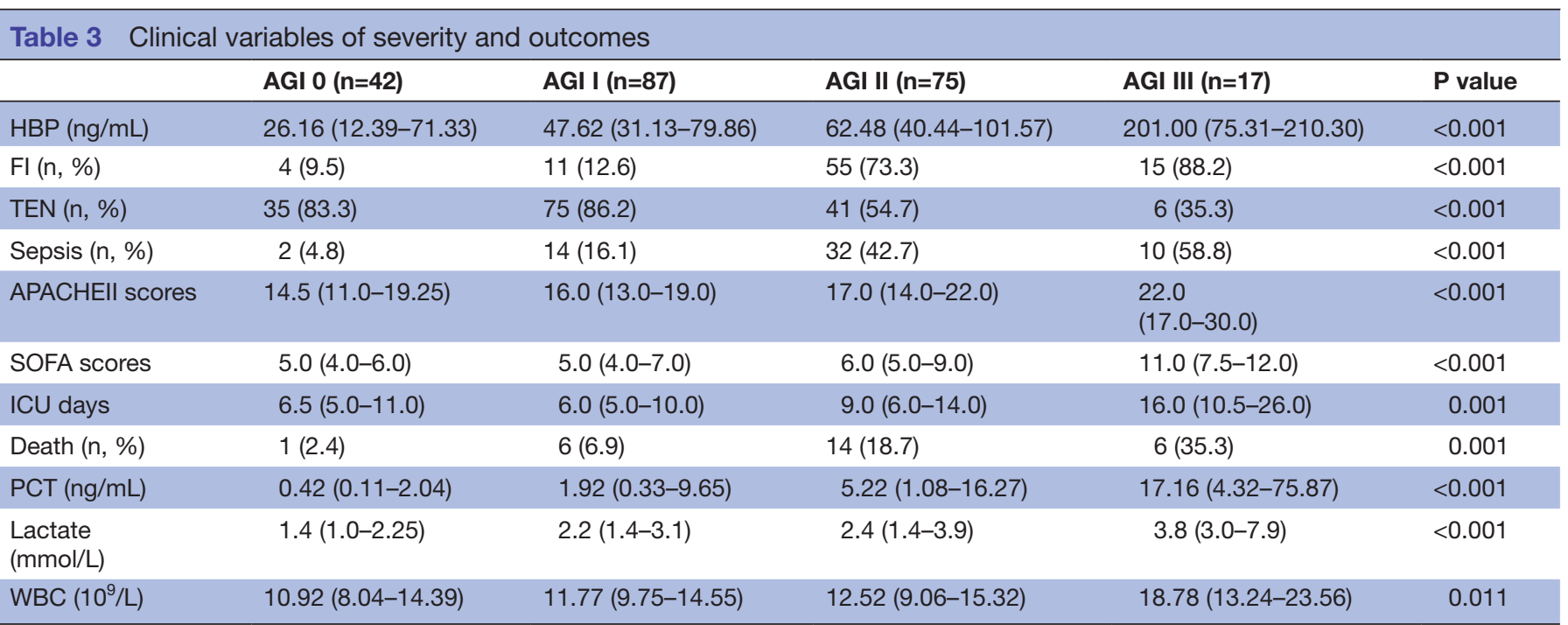

The AGI grades were summary variables for 5 days (the worst values). The HBP was summary variable for 5 days (mean values). The FI, TEN and sepsis were summary variables for 5 days. The APACHE II scores, SOFA scores, PCT, lactate and WBC were admission variables. The ICU days and death (28-day mortality) were summary variables for hospital stay.

AGI, acute gastrointestinal injury; APACHEII, acute physiology and chronic health evaluation II; FI, feeding intolerance ; HBP, heparin-binding protein; ICU, intensive care unit; PCT, procalcitonin; SOFA, sequential organ failure assessment; TEN, total enteral nutrition; WBC, white blood cell. 
Table 4 Independent factors associated with severe AGI (grade II or above) in multivariate logistic regression analysis

\begin{tabular}{llll}
\hline & OR & 95\% Cl & P value \\
\hline HBP & 1.007 & 1.001 to 1.013 & 0.024 \\
Sepsis & 2.757 & 1.235 to 6.153 & 0.013 \\
Age & 1.033 & 1.009 to 1.057 & 0.007 \\
Lactate & 1.247 & 1.024 to 1.519 & 0.028 \\
\hline
\end{tabular}

AGI, acute gastrointestinal injury; HBP, heparin-binding protein.

the ROC curves. The optimal cut-off point for TEN was $55.0 \mathrm{ng} / \mathrm{mL}$; the sensitivity was $71.9 \%$ and the specificity was $62.4 \%$. The optimal cut-off point for FI was $48.28 \mathrm{ng}$ / $\mathrm{mL}$; the sensitivity was $64.7 \%$ and the specificity was $56.6 \%$.

In this study, we defined the worst AGI grade 0 to I as mild AGI and the worst AGI grade II to III as severe AGI. The ROC curve was also used to assess the associations between HBP and AGI deterioration or severe AGI. The AUCs of AGI deterioration and severe AGI were 0.738 $(\mathrm{p}=0.001)$ and $0.774(\mathrm{p}<0.001)$, respectively. The optimal cut-off point for AGI deterioration was $53.27 \mathrm{ng} / \mathrm{mL}$; the sensitivity was $63.8 \%$ and the specificity was $57.9 \%$. The optimal cut-off point for severe AGI was $41.26 \mathrm{ng} / \mathrm{mL}$; the sensitivity was $77.2 \%$ and the specificity was $52.7 \%$. These results indicated that HBP levels were correlated with AGI grades, TEN ratios and FI incidence rates; in other words, HBP value was associated with the development of severe AGI.

\section{DISCUSSION}

This clinical retrospective study investigated the correlations between plasma HBP and GI dysfunction in critically ill patients. We found that patients with higher HBP levels had higher AGI deterioration ratios, FI incidence rates and lower TEN ratios within 5 days after admission. Patients with more severe AGI also had higher HBP levels, worse clinical severity and poorer outcomes. In other words, HBP value was associated with the development of severe AGI (grade II or above).

AGI was common in critically ill patients and could increase the risk of mortality. ${ }^{12} 14$ GI injury was often


Figure 2 The correlations of $\mathrm{HBP}$ with $\mathrm{PCT}(\mathrm{A})$, lactate $(\mathrm{B})$ and WBC (C). The HBP levels were positively correlated with (A) PCT $\left(R^{2}=0.172\right)$ and $(B)$ lactate $\left(R^{2}=0.118\right)$. (C) There was a low correlation between HBP and WBC $\left(R^{2}=0.09\right)$. HBP, heparin-binding protein; PCT, procalcitonin; WBC, white blood cell. caused by an inflammatory reaction, infection or sepsis, severe trauma, shock, pancreatitis and other critical diseases. ${ }^{14}{ }^{15}$ Accompanying by that, intestinal epithelial cell damaged, mucosal permeability increased, intestinal flora translocated and then intestinal original infection and MODS developed. ${ }^{16}$ Therefore, GI dysfunction is considered as 'motor of multiple organ failure', and it is crucial to assess GI function as early and accurately as possible. In 2012, the WGAP of the ESICM proposed the definitions and a grading system for AGI in intensive care patients. ${ }^{3} \mathrm{Hu}$ et $a l^{2}$ found that severity of the AGI grade was a predictor of all-cause mortality in critically ill patients. However, the AGI grading definitions were mainly based on clinical symptoms, and rare reports were published about objective markers with fine correlation with this AGI system. In this study, we demonstrated that plasma HBP levels were correlated with AGI grades. Moreover, our results indicated that HBP levels were correlated with TEN ratios and FI incidence rates. These findings suggested that HBP could be an objective and sensitive marker that adequately reflects GI function in widespread clinical use. However, the underlying mechanisms were unclear.

The neutrophil-derived HBP was proven to be a novel biomarker for predicting organ dysfunction in critically ill patients. ${ }^{4}$ In 2012, Linder et al proposed that HBP was associated with disease severity and increased risk of death. Tverring et at reported that HBP improved the prediction of sepsis-related AKI in their prospective, observational, multicentre study. Another prospective, observational study performed by Tydén $e t$ al found that increased plasma levels of HBP on admission to ICU were associated with respiratory and circulatory failure. ${ }^{6}$ The vascular leak and oedema formation caused by plasma HBP were considered to be the main mechanisms of the development of organ dysfunction. ${ }^{45} 17$ Endothelial cells were the initial targets of neutrophil-derived HBP released from secretory vesicles. ${ }^{4} 8$ HBP binds to glycosaminoglycan moieties of endothelium cell surface proteoglycans and impairs the endothelial barrier, leading to vascular leakage and tissue oedema. ${ }^{4516}$

However, no studies have explored the associations between HBP levels and the function of another important organ, the GI tract. HBP was not only a biomarker of infection but also a pathogenic factor for organ dysfunction in critically ill patients. ${ }^{4}$ Therefore, we hypothesised that HBP levels were also increased in enterogenic infections, and the massive amount of HBP released lead to injury of vascular endothelial cells in intestinal mucosa. Then, endothelial barriers were damaged, mucosal permeability was increased and the intestinal flora were translocated. As a result, the infection was aggravated by feedback, and the oedema and GI injury occurred. Our results may serve as an argument for this hypothesis and proposed originally that HBP levels were associated with GI dysfunction.

FI was a general term indicating intolerance of enteral feeding for any clinical reason, and FI was also correlated 
with clinical severity and adverse outcomes. ${ }^{2314} \mathrm{Hu}$ et $a l^{2}$ reported that FI incidence was approximately $24 \%$ during the first week of ICU stay, and persistent FI was an independent risk factor for mortality in critically ill patients. In the present study, we found that patients with higher HBP levels had higher FI incidence rates and lower TEN ratios during enteral feeding. Moreover, the HBP levels of patients with FI or TEN were also significantly different from those of patients without FI or TEN. The AUCs of the ROC curves also indicated that HBP had significant correlations with TEN ratio and FI. Previous studies showed that the optimal cut-off level for HBP in diagnosing sepsis, severe sepsis or septic shock was $\geq 15 \mathrm{ng}$ / $\mathrm{mL}, \geq 28.1 \mathrm{or} \quad 30 \mathrm{ng} / \mathrm{mL}$ and $\geq 103.5 \mathrm{ng} / \mathrm{mL}$, respectively. ${ }^{812} 13$ However, to our knowledge, there may be no investigations on the relations between HBP levels and GI function in critically ill patients. Our study reported that the optimal cut-off point for severe AGI was $41.26 \mathrm{ng}$ / $\mathrm{mL}$ with a sensitivity of $77.2 \%$ and a specificity of $52.7 \%$, and for FI it was $48.28 \mathrm{ng} / \mathrm{mL}$ with a sensitivity of $64.7 \%$ and a specificity of $56.6 \%$. These results indicated that we should be alert for development of AGI if the HBP value was higher than about $40 \mathrm{ng} / \mathrm{mL}$.

Citrulline or intestinal fatty acid binding protein (I-FABP) was also proposed as possible biomarker for GI function. ${ }^{18}{ }^{19}$ Citrulline level was found to be related to enterocyte function, whereas I-FABP level was found to be related to intestinal ischaemic injury. ${ }^{18}{ }^{19}$ However, the plasma concentration of the two markers were susceptible to dysfunction of liver or kidney, ${ }^{18} 19$ which may restrict their application value in critically ill patients. Therefore, recommendations of the WGAP in ESICM indicated that their clinical use in diagnosis of GI dysfunction was still unclear. ${ }^{3}$ Our findings of HBP might provide a new biomarker for GI function in critically ill patients.

The relations between HBP levels and clinical severity markers and outcomes were also studied. Kahn et al ${ }^{12}$ found that HBP demonstrated good prognostic and discriminatory properties in detecting the most severely ill patients with infection in the emergency department. Zhou $e a^{13}$ revealed that a high level of HBP in plasma was associated with sepsis, which might be a useful diagnostic marker in patients with suspected sepsis. In this study, the APACHEII score, plasma PCT, WBC and lactate were used as indicators of disease severity, and we found that HBP was positively correlated with PCT and lactate within 5 days after ICU admission. Moreover, sepsis incidence, ICU days and 28-day mortality were used as indicators of clinical outcomes, and we found that increased HBP was positively correlated with sepsis. The AUCs of the ROC curves also suggested this phenomenon. Our results were also mainly consistent with previous reports.

There are no uniform specific conditions to perform the HBP testing. In our centre, patients with inflammation, infection, sepsis, trauma, burn, hypotension, shock and organ dysfunction were generally considered for HBP measurements. Also 116 elective surgery patients met these conditions and received HBP tested twice or more within 5 days of ICU, and they were included in our study. However, this was a minority of surgical patients treated in our ICU during the study period (see figure 1 ), as 2816 patients with ICU stay $<5$ days and 411 patients with HBP tested only once were excluded.

Some limitations of this study should be discussed. Since HBP is derived from neutrophil cytoplasm, patients with agranulocytosis may not be suitable for HBP testing. ${ }^{4}$ Neutrophils have a short life and could be activated in vitro; therefore, the blood sample should be centrifuged within 1-2 hours and then refrigerated or frozen as soon as possible. ${ }^{45}$ Due to the limitations of our study cohort (mainly surgery patients), single-centre retrospective design and small sample size, the results might not be applicable to all critically ill patients, and the accuracy should be confirmed by large-scale clinical prospective studies. Moreover, since the study was not based on pathophysiological models, and the results were hypothesis generating, the exact mechanisms of HBP on GI function should be tested by more basic experiments. In addition, since no patients with AGI grade IV were included in this study, the conclusions might not be applicable to all critically ill patients. Finally, since some parameters were only recorded once within 5 days, the later effects of HBP on GI function should also be tested by future randomised controlled clinical trials.

\section{CONCLUSIONS}

Our retrospective clinical study showed that HBP levels were associated with GI dysfunction. Increased HBP was positively correlated with sepsis but it was not correlated with 28-day mortality. More basic experiments and clinical prospective trials are needed to verify our results.

Acknowledgements The authors thank Qiao Liu for her assistance in the statistical analysis of this study. The authors also thank Yuan ST, Chen YM, Xu QL and Wang S of Department of Intensive Care Unit at Nanjing First Hospital for their contributions to this study. In addition, J-KS and his family especially thank X-PS for her meticulous care and support during the past 10 years.

Contributors J-KS, XS, X-PS, XW, Q-KS and X-WM designed the research; J-KS, $\mathrm{XS}$ and $\mathrm{W}-\mathrm{HZ}$ performed the research; J-KS, XS and W-HZ analysed the data; J-KS and X-PS wrote the paper.

Funding This study was supported by the National Natural Science Foundation of China (No. 81701881, 81801891) and the Nanjing Medical Science and Technology Development Foundation (No. YKK17102, YKK18108).

Competing interests None declared.

Patient and public involvement Patients and/or the public were not involved in the design, or conduct, or reporting, or dissemination plans of this research.

Patient consent for publication Not required.

Ethics approval The study was reviewed and approved by the institutional review board of Nanjing First Hospital, whereas written informed consent was waived because this was a retrospective study.

Provenance and peer review Not commissioned; externally peer reviewed. Data availability statement No data are available.

Open access This is an open access article distributed in accordance with the Creative Commons Attribution Non Commercial (CC BY-NC 4.0) license, which permits others to distribute, remix, adapt, build upon this work non-commercially, and license their derivative works on different terms, provided the original work is 
properly cited, appropriate credit is given, any changes made indicated, and the use is non-commercial. See: http://creativecommons.org/licenses/by-nc/4.0/.

\section{ORCID iD}

Qian-Kun Shi http://orcid.org/0000-0003-4193-9754

\section{REFERENCES}

1 Zhang D, Li Y, Ding L, et al. Prevalence and outcome of acute gastrointestinal injury in critically ill patients: a systematic review and meta-analysis. Medicine 2018;97:e12970.

$2 \mathrm{Hu} \mathrm{B}$, Sun R, Wu A, et al. Severity of acute gastrointestinal injury grade is a predictor of all-cause mortality in critically ill patients: a multicenter, prospective, observational study. Crit Care 2017;21:188.

3 Reintam Blaser A, Malbrain MLNG, Starkopf J, et al. Gastrointestinal function in intensive care patients: terminology, definitions and management. recommendations of the ESICM Working group on abdominal problems. Intensive Care Med 2012;38:384-94.

4 Fisher J, Linder A. Heparin-binding protein: a key player in the pathophysiology of organ dysfunction in sepsis. J Intern Med 2017;281:562-74.

5 Tverring J, Vaara ST, Fisher J, et al. Heparin-binding protein (HBP) improves prediction of sepsis-related acute kidney injury. Ann Intensive Care 2017;7:105.

6 Tydén J, Herwald H, Sjöberg F, et al. Increased plasma levels of heparin-binding protein on admission to intensive care are associated with respiratory and circulatory failure. PLoS One 2016;11:e0152035.

7 Lin Q, Shen J, Shen L, et al. Increased plasma levels of heparinbinding protein in patients with acute respiratory distress syndrome. Crit Care 2013;17:R155.

8 Linder A, Åkesson P, Inghammar M, et al. Elevated plasma levels of heparin-binding protein in intensive care unit patients with severe sepsis and septic shock. Crit Care 2012;16:R90.
9 Rhodes A, Evans LE, Alhazzani W, et al. Surviving sepsis campaign: international guidelines for management of sepsis and septic shock: 2016. Crit Care Med 2017;45:486-552.

10 ARDS Definition Task Force, Ranieri VM, Rubenfeld GD, et al. Acute respiratory distress syndrome: the Berlin definition. JAMA 2012;307:2526-33.

11 Kellum JA, Lameire N, KDIGO AKI Guideline Work Group. Diagnosis, evaluation, and management of acute kidney injury: a KDIGO summary (Part 1). Crit Care 2013;17:204.

12 Kahn F, Tverring J, Mellhammar L, et al. Heparin-binding protein as a prognostic biomarker of sepsis and disease severity at the emergency department. Shock 2019;52:e135-45.

13 Zhou Y, Liu Z, Huang J, et al. Usefulness of the heparin-binding protein level to diagnose sepsis and septic shock according to Sepsis-3 compared with procalcitonin and $C$ reactive protein: a prospective cohort study in China. BMJ Open 2019;9:e026527.

14 Li H, Zhang D, Wang Y, et al. Association between acute gastrointestinal injury grading system and disease severity and prognosis in critically ill patients: a multicenter, prospective, observational study in China. J Crit Care 2016;36:24-8.

15 Zhang D, Fu R, Li Y, et al. Comparison of the clinical characteristics and prognosis of primary versus secondary acute gastrointestinal injury in critically ill patients. J Intensive Care 2017;5:26.

16 Sun J-K, Zhang W-H, Chen W-X, et al. Effects of early enteral nutrition on Th17/Treg cells and IL-23/IL-17 in septic patients. World $J$ Gastroenterol 2019;25:2799-808.

17 Fisher J, Russell JA, Bentzer P, et al. Heparin-binding protein (HBP): a causative marker and potential target for heparin treatment of human sepsis-induced acute kidney injury. Shock 2017;48:313-20.

18 Weimann A, Felbinger TW. Gastrointestinal dysmotility in the critically ill: a role for nutrition. Curr Opin Clin Nutr Metab Care 2016;19:353-9.

19 Blaser A, Padar M, Tang J, et al. Citrulline and intestinal fatty acidbinding protein as biomarkers for gastrointestinal dysfunction in the critically ill. Anaesthesiol Intensive Ther 2019;51:230-9. 